\title{
Un dialogue nécessaire dans le monde du soin
}

\author{
Marc-Antoine Bornet ${ }^{a}$, Etienne Rochat ${ }^{b}$ \\ a MMed, Médecin assistant, Doctorant à la Plateforme Médecine, Spiritualité, Soins et Société (MS3), CHUV \\ ${ }^{\mathrm{b}} \mathrm{MTh}$, Accompagnant spirituel, Responsable de la Plateforme Médecine, Spiritualité, Soins et Société (MS3), CHUV
}

\section{Sa Sainteté le $17^{\mathrm{e}}$ Karmapa à l'Université de Lausanne}

En mai dernier, trois ans après la venue du Dalaï Lama, l'Université de Lausanne a accueilli le $17^{\mathrm{e}}$ Gyalwang Karmapa. Cette éminente figure du bouddhisme tibétain a dialogué avec les étudiants en médecine de première année, dans le cadre de leur cours d'éthique. En introduction, le Professeur Lazare Benaroyo souligne que cet événement est une occasion unique offerte aux étudiants pour approfondir leur compréhension des valeurs fondamentales du soin et pour élargir leur horizon sur des enjeux fondamentaux de l'éthique médicale. La formule choisie, sous forme d'un dialogue avec des étudiants, est singulière et stimulante.

\section{Le patient: santé et souffrance}

Première thématique: la santé et sa définition. Un étudiant cite les composantes physiques, mentales et sociales de la définition donnée par l’OMS. Une étudiante ajoute qu'elle y associe la conservation des capacités physiques et cognitives contribuant à maintenir la liberté d'action du patient. Enfin, un autre étudiant estime nécessaire de penser également à la santé de l'esprit, considérant que la dimension spirituelle doit être ajoutée à cette définition.

Le Karmapa commence par reconnaître la compréhension vaste et profonde des étudiants, malgré leur jeune âge. Pour lui, la santé de l'individu doit être considérée comme l'harmonie qui existe entre l'extériorité de la personne et son intériorité. Il rappelle qu'une personne peut vivre de grandes souffrances intérieures sans que son extérieur en soit affecté. En ce sens, le Karmapa lie aussi la santé à une forme d'équilibre émotionnel et de paix intérieure.

La question de la souffrance, notion plus complexe que la douleur, ne manque pas de soulever un débat dans l'auditoire: la souffrance peut-elle être positive? Ne serait-ce pas plutôt les efforts pour y faire face qui sont positifs et qui nous font grandir?

La philosophie bouddhiste en distingue trois formes: la souffrance de la souffrance (évidente, une sensation de souffrance souvent liée à la douleur), la souffrance du changement (causée par le changement et l'impermanence d'états heureux) et la souffrance naturelle (tout bonheur dépendant de quelque chose, la vie est considérée comme souffrance). Pour le Karmapa, la souffrance est parfois liée au bonheur, dans le sens où le bonheur peut être induit par un moment de répit dans la souffrance ou la difficulté. Le porteur d'une lourde charge sur l'épaule gauche, qui la laisse tomber et la reprend sur l'épaule droite éprouvera un soulagement. Ainsi, tout bonheur résulterait d'un événement passé.

\section{La relation: quand la compassion est à la source du soin}

Question soulevée par le Docteur Raphaël Bonvin, «Que signifie prendre soin des patients?» Cette nouvelle interrogation reçoit un grand nombre de réponses: viser à améliorer la santé physique; accompagner les patients, les aider à retrouver leur équilibre; les respecter dans leur fragilité sans émettre de jugement.

Pour le Karmapa, l'aspect principal du "prendre soin» n'est pas l'aspect technique, mais le désir profond du bonheur d'autrui. Preuve en est qu'il considère aussi devoir prendre soin des personnes venant le rencontrer, alors qu'il n'a pas de formation de médecin. Le soin doit se baser sur la compassion, valeur qui soustend et alimente l'empathie, capacité à comprendre et à entrer dans le monde de l'autre. Cette mise en œuvre de la compassion permet à l'être souffrant de ne pas se sentir seul et de conserver de l'espérance, même dans les moments les plus difficiles. En rapprochant le médecin du patient, la compassion ouvre à une meilleure compréhension de l'autre.

\section{Le médecin: confronté à la mort - et à sa vie?}

Il s'avère toujours difficile de donner un conseil au médecin confronté au doute dans ses prises de décisions, et le Karmapa dit sa difficulté à répondre. Lui aussi se 


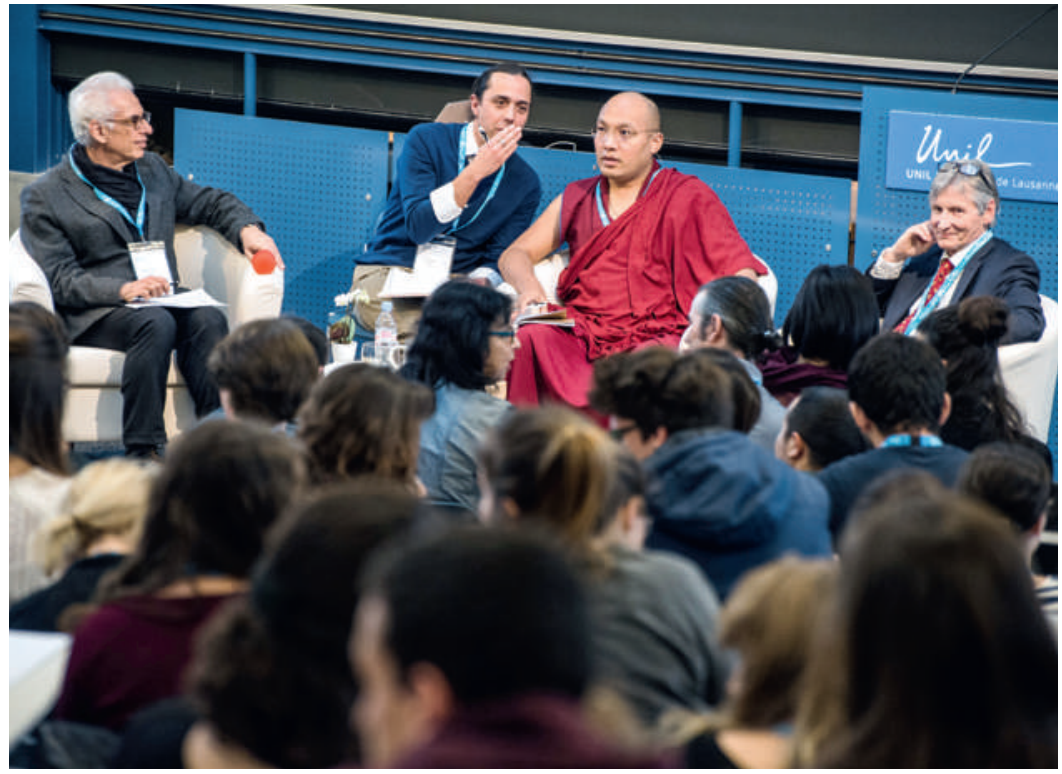

Le $17^{\circ}$ Karmapa dialogue avec des étudiants en médecine à I'Université de Lausanne, le 23 mai 2016.
Correspondance:

Marc-Antoine Bornet

CHUV

Rue du Bugnon 46

CH-1011 Lausanne

marc-antoine.bornet[at]

chuv.ch sent dépourvu face à certaines questions posées par les fidèles venus le consulter.

Il aborde aussi son vécu face à la mort, à l'angoisse de partir avant d'avoir accompli ce qu'il aurait dû, ou avant d'avoir pu s'y préparer. Pour lui, la mort n'est pas une fin, c'est une vie nouvelle qui commence. De cette réflexion et vision sur la mort découlent de fortes exigences dans la conduite existentielle de chacune et chacun, notamment pour faire naître un sentiment d'accomplissement dans la vie, sorte de bouclier contre l'angoisse de la mort.

Comment réussir à prendre soin de soi-même et à trouver son propre équilibre? Et surtout comment intégrer les expériences à la fois extraordinaires et terribles vécues au contact quotidien des patients? Les intervenants, sans la nommer, touchent à la notion de croissance personnelle ("personal growth»), capacité de découvrir en soi des ressources considérables.

\section{Le cadre: définir les priorités}

Une personnalité du domaine médical, touchée par les propos humanistes tenus par ces jeunes étudiants, rappelle que ces échanges contrastent avec ce qu'elle constate quelques années plus tard à leur début en clinique, au moment où les jeunes médecins sont confrontés à une très importante technicité.

Comment entretenir et maintenir cette capacité réflexive et ce désir de grande proximité avec le patient, une fois engagé dans une formation et un environnement professionnel qui exigent performance et efficacité? Aujourd'hui, le vœu des Universités - et cet évènement en est une belle preuve - est d'offrir une formation de plus en plus élargie en vue de répondre aux défis humains de la pratique future. Le Professeur Philippe Moreillon rappelle d'ailleurs que la formation médicale actuelle souhaite développer encore plus le côté humain, notamment pour ceux qui seront les médecins de demain.

\section{Ethique et spiritualité: le nécessaire dialogue}

Cependant, cette préoccupation risque de rester vaine si les modèles de prise en charge des patients n'autorisent pas, dans l'hôpital comme au cabinet, l'intégration des questions existentielles d'une part et des questions d'éthique de la vulnérabilité qu'elles suscitent obligatoirement d'autre part.

Cette éthique, même si elle reste séculière et non inféodée à un dogme, s'enrichit d'être ouverte à la dimension spirituelle du patient.

De plus, et ce dialogue avec le Karmapa l'a bien montré, la pratique de cette éthique comporte aussi une invitation à découvrir soi-même sa propre spiritualité. Autrement dit, connaître ses valeurs et ce qui donne du sens à sa vie est un savoir nécessaire pour accueillir et accompagner la souffrance du patient.

\section{Crédit photo}

Felix Imhof, (c) UNIL 